\title{
ON THE COVARIANCE OF THE COMMUNITY MATRIX ${ }^{1}$
}

\author{
JOHN H. VANDERMEER \\ Department of Zoology, University of Michigan, Ann Arbor 48104
}

\begin{abstract}
A clarification of the meaning of the covariance of the community matrix is presented along with calculating equations for both the mean species covariance and the covariance of the row and column means. A brief discussion of $\alpha$ selection and its determination of the covariance is given, followed by tables for determining the expected number of species in a community from a knowledge of mean $\alpha$ and $\operatorname{cov} \alpha$.
\end{abstract}

Much interest in community ecology seems to have been generated by the theoretical constructs surrounding the community matrix (Levins 1968, Vandermeer 1970). Culver (1970), for example, uses the community matrix to investigate the nature of interference and exploitative competition in a community of cave isopods. Culver also points out the fact that the methods formerly used to estimate the covariance of the community matrix lead to the uneasy result that the covariance changes depending upon how the species are ordered.

In the present communication I derive a form for the covariance that is in accord with the original expectation of the expansion of the determinant of the community matrix. The actual computation of this form does not result in a value that changes with a changing ordering of the species, and furthermore, the interpretation of this covariance is much more easily couched in biological language than the former concept was.

The derivation presented by me (Vandermeer 1970) and by Levins (1968) of the expected value of the determinant depended upon the following general relationship,

$$
\mathrm{E}\left(\alpha_{i j} \alpha_{j i}\right)=\bar{\alpha}^{2}+\operatorname{cov}\left(\alpha_{j j} \alpha_{j i}\right),
$$

which is correct given a proper interpretation of $\operatorname{cov}\left(\alpha_{i j} \alpha_{j i}\right)$. Throughout this paper $\alpha_{i j}$ refers to the effect of species $j$ on $i$ and $m$ refers to the number of species in the community. To see this interpretation, take expectations with respect to one index variable at a time. That is, the expected value of $\alpha_{i j} \alpha_{j i}$ with respect to $j$ is $\bar{\alpha}_{i} \cdot \bar{\alpha}_{\cdot i}+\operatorname{cov} \alpha_{i} \cdot \alpha_{\cdot i}$. Taking the expectation of that result with respect to $i$, we obtain

$$
\mathrm{E}\left(\alpha_{i j} \alpha_{j i}\right)=\bar{\alpha}^{2}+\left[\overline{\operatorname{cov} \alpha_{i .} \alpha_{. i}}+\operatorname{cov} \vec{\alpha}_{i,} \bar{\alpha}_{. i}\right]
$$

where the term in the brackets corresponds to what Levins and $I$ have referred to as the covariance of the community matrix $\left[\operatorname{cov} \alpha\right.$ or $\left.\operatorname{cov}\left(\alpha_{i j} \alpha_{j i}\right)\right]$.

For purposes of computation the following formulae are useful:

$\overline{\operatorname{cov} \alpha_{i .} \alpha_{. i}}=\sum_{i=1}^{m}\left\{\frac{\sum_{i \neq i} \alpha_{i j} \alpha_{j i}-\left(\sum_{j \neq i} \alpha_{j i} \sum_{j \neq i} \alpha_{i j}\right) /(m-1)}{m(m-2)}\right\}$

${ }^{1}$ Received May 20, 1971; accepted September 20, 1971. $\operatorname{cov} \bar{\alpha}_{i .} \bar{\alpha}_{. i}=\frac{\sum_{i=1}^{m} \bar{\alpha}_{i .} \bar{\alpha}_{. i}-\left(\sum_{i=1}^{m} \bar{\alpha}_{i .} \sum_{i=1}^{m} \bar{\alpha}_{. i}\right) / m}{m-1}$

where $\bar{\alpha}_{i .}=\sum_{j \neq i} \alpha_{i j} /(m-1)$

and $\bar{\alpha}_{\cdot i} \sum_{j \neq i} \alpha_{j i} /(m-1)$.

The above equations are written under the assumptions that the community matrix in question is actually a representation of a sample of $m$ species from a larger community. Thus we obtain the usual degrees of freedom $(m-2)$ in equation (1) and $(m-1)$ in equation (2). If, however, the community matrix in question is a representation of an entire community rather than a sample thereof, the point from which the deviations in the covariance are measured is no longer a sample statistic (mean $\alpha$ ) but rather a true parametric value. Thus, if we are concerned with a community matrix that includes all of the species in some community, the term $(m-2)$ in equation (1) should be changed to $(m-1)$ and the term $(m-1)$ in equation (2) should be changed to $m$.

Considering each term separately we first consider $\operatorname{cov} \bar{\alpha}_{i .} \bar{\alpha}_{i, i}$. Note that $\bar{\alpha}_{i}$. refers to the mean value of the $\alpha$ 's in the $i$ th column, or the mean interactive effect perpetrated on the community as a whole by the $i$ th species. Call this value the "species effect." A species that exhibits a large effect on most of the other species in the community is said to have a large species effect. On the other hand, $\bar{\alpha}_{. i}$ is the mean value of the $\alpha$ 's in the $i$ th row, or the mean interactive effect perpetrated on the $i$ th species by the community as a whole. Call this value the "community effect." A species that manages more or less to avoid the interactive effects of most of the other species in the community has a low community effect. Thus we have the species effect, which is the mean value of $\alpha$ in a particular column of the community matrix, and the community effect, which is the mean value of $\alpha$ in a particular row of the community matrix.

A great deal of information about the structure of the community is available from a direct examination of the row and column means, but for this paper 
we are interested in the covariance between them. Intuitively, if one can easily predict the value of the community effect from a knowledge of the species effect, the absolute value of $\operatorname{cov} \bar{\alpha}_{i} \bar{\alpha}_{. i}$ will be high. Specifically, if a large species effect implies a large community effect and a small species effect implies a small community effect, $\operatorname{cov} \bar{\alpha}_{i,} \bar{\alpha}_{, i}$ will be large and positive. On the other hand, if a large species effect implies a small community effect, $\operatorname{cov} \bar{\alpha}_{i .} \bar{\alpha}_{. i}$ will be large and negative. If it is impossible to say anything about the species effect from a knowledge of the community effect, $\operatorname{cov} \bar{\alpha}_{i .} \vec{\alpha}_{. i}$ is near zero.

Next consider the term $\overline{\operatorname{cov} \alpha_{i .} \alpha_{. i}}$. Each species has a particular value of $\operatorname{cov} \alpha_{i} \alpha_{. i}$, i.e., the "species covariance" for the $i$ th species. If a large $\alpha_{i j}$ implies a large $\alpha_{j i}$ for all values of $j$, the species covariance for the $i$ th species is large and positive. If a large $\alpha_{i j}$ implies a small $\alpha_{j i}$ for all values of $j$, the species covariance is large and negative. If it is not possible to predict the value of $\alpha_{j i}$ from a knowledge of $\alpha_{i j}$, the species covariance is near zero. The quantity $\overline{\operatorname{cov} \alpha_{i .} \alpha_{. i}}$ is obviously the mean value of all the species covariances.

The computation of $\operatorname{cov} \bar{\alpha}_{i,} \bar{\alpha}_{, i}$ and $\overline{\operatorname{cov} \alpha_{i .} \alpha_{, i}}$ is illustrated in the following example. Consider the community matrix presented by Culver (1970), where his negative values have been replaced by zeros and all $\alpha$ 's have been rounded to one decimal place. Thus we have the following community matrix:

$$
\begin{array}{|llll}
1 & 0 & .1 & 0 \\
0 & 1 & 1.1 & .6 \\
.1 & .8 & 1 & .4 \\
.1 & .8 & .8 & 1
\end{array} \mid
$$

The species effects (row means, deleting principal diagonal) are $.03, .57, .43, .57$, and the community effects (column means, deleting principal diagonal) are $.07, .53, .67, .33$. The sum of the species effects is 1.60 , the sum of the community effects is 1.60 , and the sum of the cross products is .78. Thus the final marginal covariance $\left(\operatorname{cov} \bar{\alpha}_{i .} \bar{\alpha}_{. i}\right)$ is

$$
[.78-(1.60)(1.60) / 4] / 3=.047 \text {. }
$$

It is next necessary to compute a species covariance for each species and take the average of the species covariances. Consider, for example, species number 2 in the above community matrix. We are concerned with the second row and second column of the matrix, namely $0,1,1.1, .6$, and $0,1, .8, .8$. Because of the original derivation of the expected value of the determinant we must ignore the 1's in the principle diagonal, making the row and column of concern $0,1.1, .6$ and $0, .8, .8$. The row sum is 1.7 , the column sum is 1.6 , and the sum of products is 1.36. Thus the species covariance for the second species is $[1.36-(1.7)(1.6) / 3] / 2=.225$. Performing this operation for all four species in the community, we obtain $\operatorname{cov} \alpha_{1}, \alpha_{.1}=.047, \operatorname{cov} \alpha_{2 .} \alpha_{.2}=.225$, $\operatorname{cov} \alpha_{3 .} \alpha_{.3}=.170$, and $\operatorname{cov} \alpha_{4 .} \alpha_{.4}=.116$, which gives a mean species covariance of .149 .

The creation of methods to measure the actual interaction coefficients in any natural situation is the most difficult problem facing community matrix theory. I will not discuss that problem in this paper, but it is important to emphasize that the estimation of $\alpha$ 's is distinct from the various other features of the theory. In this paper we assume that $\alpha$ 's have been estimated and that they are correct. Given that assumption we can compute $\overline{\operatorname{cov} \bar{\alpha}_{i .} \bar{\alpha}_{. i}}, \operatorname{cov} \alpha_{i .} \alpha_{. i}$, and $\bar{\alpha}$ and determine from tabulated values what the number of species in a saturated community is expected to be. For details of how such tables are derived see Levins (1968) or Vandermeer (1970).

The table presented in Levins (1968) shows expected values for only positive covariances. It is not difficult to imagine communities with negative covariances, e.g., a large species effect implies a small community effect. To see how such a relationship could come about, consider briefly the evolution of interspecific competitive ability. Analogous to $r$ and $K$ selection (MacArthur and Wilson 1967) we now consider the processes of $\alpha$ selection. Creating an artificial dichotomy, and supposing interference competition to be negligible, a species might evolve a high competitive ability either by becoming good at escaping the effects of potential competitiors, or by becoming more efficient at utilizing a limiting resource. That is, a species could become a good competitor either by decreasing its community effect or increasing its species effect. Evolution might proceed in such a way that competition is either maximized or ignored, meaning that a large species effect implies a small community effect and $\operatorname{cov} \bar{\alpha}_{i} \bar{\alpha}_{. i}$ is large and negative. On the other hand it might be argued that in maximizing competitive ability a species must concentrate on either the species effect or the community effect. Naturally this set of circumstances results in $\operatorname{cov} \bar{\alpha}_{i,} \bar{\alpha}_{i i}$ being large and positive.

The above is not meant to be an exhaustive treatment of $\alpha$ selection but merely to show that the covariance is expected to be either positive or negative. In fact, examples computed from nature include both positive and negative covariances.

In light of the above, Tables 1 and 2 are presented as potentially useful extensions of the original table presented by Levins. The row headings of Tables 1 and 2 are the various values of $\operatorname{cov} \alpha$ where $\operatorname{cov} \alpha=$ $\operatorname{cov} \bar{\alpha}_{i .} \bar{\alpha}_{. i}+\overline{\operatorname{cov} \alpha_{i .} \alpha_{i}}$, and the column headings are the values of $\bar{\alpha}$. In computing Tables 1 and 2, a great deal of round-off error frequently led to expected numbers of species clearly too large or too small to 
TABle 1. The expected number of species in a community as a function of $\bar{\alpha}$ (columns) and cov $\alpha$ (rows), for positive covariances

\begin{tabular}{|c|c|c|c|c|c|c|c|c|c|c|c|c|c|c|c|c|}
\hline $\operatorname{cov} \alpha$ & $\bar{\alpha}$ & .0125 & .025 & .0375 & .0625 & .0875 & .0925 & .0975 & .105 & .120 & .140 & .160 & 20 & .40 & .60 & .80 \\
\hline $\begin{array}{l}.003 \\
.006 \\
.009 \\
.012 \\
.015 \\
.018 \\
.021 \\
.024 \\
.030 \\
.036 \\
.048 \\
.084 \\
.120\end{array}$ & & $\begin{array}{r}67 \\
44 \\
33 \\
26 \\
22 \\
19 \\
16 \\
15 \\
12 \\
11 \\
9 \\
6 \\
5\end{array}$ & $\begin{array}{r}65 \\
43 \\
32 \\
26 \\
21 \\
18 \\
16 \\
15 \\
12 \\
11 \\
9 \\
6 \\
5\end{array}$ & $\begin{array}{r}58 \\
42 \\
32 \\
25 \\
21 \\
18 \\
16 \\
14 \\
12 \\
11 \\
9 \\
6 \\
5\end{array}$ & $\begin{array}{r}44 \\
40 \\
30 \\
24 \\
20 \\
18 \\
16 \\
14 \\
12 \\
10 \\
8 \\
6 \\
4\end{array}$ & $\begin{array}{r}35 \\
34 \\
30 \\
23 \\
19 \\
17 \\
15 \\
13 \\
11 \\
10 \\
8 \\
6 \\
4\end{array}$ & $\begin{array}{r}34 \\
32 \\
28 \\
23 \\
19 \\
17 \\
15 \\
13 \\
11 \\
10 \\
8 \\
6 \\
4\end{array}$ & $\begin{array}{r}32 \\
32 \\
27 \\
23 \\
19 \\
17 \\
15 \\
13 \\
11 \\
10 \\
8 \\
5 \\
4\end{array}$ & $\begin{array}{r}30 \\
28 \\
28 \\
23 \\
19 \\
16 \\
15 \\
13 \\
11 \\
10 \\
8 \\
5 \\
4\end{array}$ & $\begin{array}{r}28 \\
27 \\
26 \\
22 \\
18 \\
16 \\
14 \\
13 \\
11 \\
10 \\
8 \\
5 \\
4\end{array}$ & $\begin{array}{r}27 \\
24 \\
24 \\
21 \\
18 \\
15 \\
14 \\
12 \\
11 \\
9 \\
8 \\
5 \\
4\end{array}$ & $\begin{array}{r}24 \\
22 \\
22 \\
20 \\
17 \\
15 \\
13 \\
12 \\
10 \\
9 \\
7 \\
5 \\
4\end{array}$ & $\begin{array}{r}22 \\
20 \\
21 \\
19 \\
16 \\
14 \\
12 \\
11 \\
10 \\
8 \\
7 \\
5 \\
4\end{array}$ & $\begin{array}{r}15 \\
15 \\
13 \\
13 \\
10 \\
9 \\
8 \\
8 \\
7 \\
6 \\
5 \\
4 \\
3\end{array}$ & $\begin{array}{r}11 \\
11 \\
8 \\
7 \\
6 \\
6 \\
5 \\
5 \\
4 \\
4 \\
4 \\
3 \\
2\end{array}$ & $\begin{array}{l}6 \\
5 \\
4 \\
4 \\
3 \\
3 \\
3 \\
3 \\
3 \\
2 \\
2 \\
2 \\
2\end{array}$ \\
\hline
\end{tabular}

Table 2. The expected number of species in a community as a function of $\bar{\alpha}$ (columns) and cov $\alpha$ (rows), for negative covariances

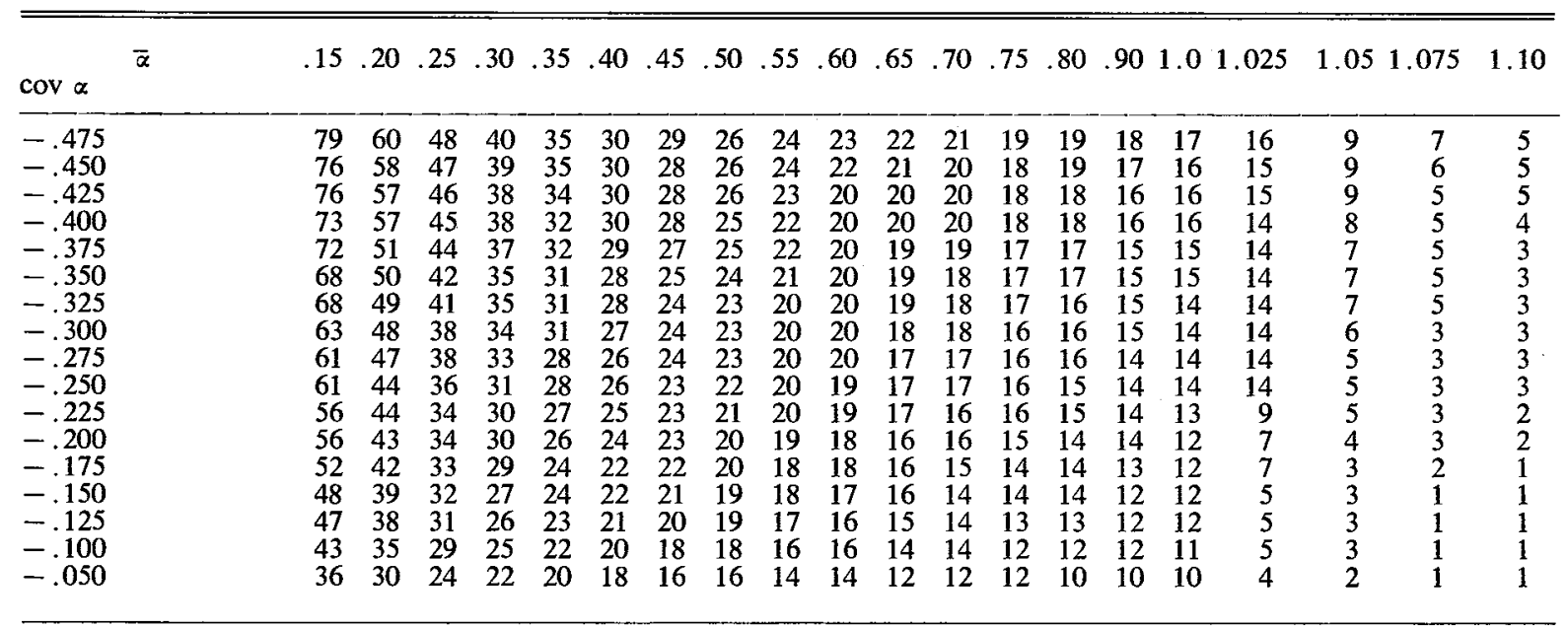

fit into the mode of the table. In such cases, $\bar{\alpha}$ and $\operatorname{cov} \alpha$ were changed very slightly and a new expected number of species computed. This "round-off" procedure results in a monotonically decreasing number of species in each row and column of Tables 1 and 2.

\section{ACKNOWLEDGMENTS}

I wish to thank Dave Culver for his helpful comments and an annonymous reviewer for pointing out the prob- lem with the degrees of freedom in the calculating equations for the covariances.

\section{Literature Cited}

Culver, D. 1970. Analysis of simple cave communities: niche separation and species packing. Ecology 51:949958.

Levins, R. 1968. Evolution in changing environments. Princeton Univ. Press, Princeton, N.J. 120 p.

Vandermeer, J. H. 1970. The community matrix and the number of species in a community. Amer. Natur. 104:73-84. 